\title{
EFECTO DEL CAMBIO CLIMÁTICO SOBRE LA FISIOLOGÍA DE LAS PLANTAS CULTIVADAS: UNA REVISIÓN
}

\section{EFFECT OF CLIMATE CHANGE ON THE PHYSIOLOGY OF CROP PLANTS: A REVIEW}

\author{
Alfredo Jarma Orozco ${ }^{1}$; Carlos Cardona Ayala²; Hermes Araméndiz Tatis ${ }^{3}$
}

\begin{abstract}
${ }^{1}$ I.A. Ph.D., Facultad de Ciencias Agrícolas, Universidad de Córdoba, Montería. ajarma24@yahoo.com; 2 I.A. M.Sc. Facultad de Ciencias Agrícolas, Universidad de Córdoba, ccardonaayala@yahoo.com; 3 I.A. Ph.D. Facultad de Ciencias Agrícolas, Universidad de Córdoba, haramendiz@hotmail.com - Ciudad Universitaria Carrera 6 No. 76-103. Código Postal: 354. Montería - Colombia
\end{abstract}

Rev. U.D.C.A Act. \& Div. Cient. 15(1): 63 - 76, 2012

\section{RESUMEN}

Para analizar los efectos del cambio climático sobre la fisiología de las plantas es necesario considerar varios factores ambientales y su interacción con los procesos fisiológicos de las plantas. El objetivo de esta revisión fue presentar el conocimiento actual de las investigaciones relacionadas con los efectos del cambio climático sobre la fisiología de las plantas. Se han abordado los efectos del incremento de $\mathrm{CO}_{2}$, temperatura, agua (déficit y exceso hídrico) y ozono, sobre el crecimiento y el desarrollo de varias especies cultivadas. El incremento de la temperatura generará problemas de erosión genética y conducirá a la extinción de muchas especies vegetales; las altas temperaturas pueden afectar adversamente la fotosíntesis, la respiración, las relaciones hídricas y la estabilidad de las membranas, la regulación hormonal y el metabolismo secundario de las plantas. La fotosíntesis neta, se incrementa progresivamente con el aumento del $\mathrm{CO}_{2}$, en particular, en especies $\mathrm{C}_{3}$, pero a altas temperaturas, se reduce por efecto del aumento en la fotorrespiración. La exposición al ozono $\left(\mathrm{O}_{3}\right)$ disminuye la difusión estomática, la tasa fotosintética, afecta la partición de asimilados y, en general, reduce el crecimiento de las plantas.

Palabras clave: Variabilidad climática, temperatura, dióxido de carbono, temperatura, agua, ozono.

\section{SUMMARY}

To analyze the effects of climate change on plant physiology is necessary to consider various environmental factors and their interaction with physiological process. The objective of this review is to present the current knowledge of research related to climate change effects on plant physiology. This review covers the effects of increased $\mathrm{CO}_{2}$, temperature, water (water deficit and excess water) and ozone on growth and development of various crop species. The temperature increase will generate problems of genetic erosion and lead to the extinction of many plant species, high temperatures can adversely affect photosynthesis, respiration, water relations and membrane stability, hormone regulation and secondary metabolism plants. Net photosynthesis increases gradually with the increase of $\mathrm{CO}_{2}$, especially in $\mathrm{C}_{3}$ species, but at high temperatures is reduced because of increased photorespiration. Exposure to ozone $\left(\mathrm{O}_{3}\right)$ reduces stomata diffusion, the photosynthetic rate, affects the partition of assimilates and generally reduces plant growth.

Key words: Climate variability, temperature, carbon dioxide, water, ozone.

\section{INTRODUCCIÓN}

Las variaciones climáticas globales han ocurrido de manera constante a lo largo de la historia de la tierra; sin embargo, en las últimas décadas, la acción del hombre ha rebasado la capacidad de cambio de los procesos naturales, transformando la atmósfera, la hidrósfera y la biósfera, con velocidades superiores a las que normalmente ocurrían. Las fluctuaciones en los elementos del clima (temperatura y precipitación) que se han presentado en los últimos años han sido objeto de diversos estudios, ya sea para su 
monitoreo, su ocurrencia, su frecuencia, su duración, su prevención, su predicción y su impacto. Particularmente, en el sector agrícola existe un marcado interés en determinar las estrategias que algunas especies utilizan para mitigar los efectos adversos del cambio climático, especialmente, en la producción de alimentos (Hunt \& Elliott, 2002; Lau et al. 2011).

El calentamiento global es un fenómeno complejo y sus impactos a gran escala son difíciles de predecir con certeza; no obstante, cada año los científicos tienen más información sobre la forma en que el calentamiento global está afectando al planeta y muchos de ellos concuerdan que es probable que algunas consecuencias ocurran si continúan las tendencias actuales. El bióxido de carbono y otros contaminantes del aire, se acumulan en la atmósfera formando una capa cada vez más gruesa, atrapando el calor del sol y causando el calentamiento del planeta (NRDC, 2008).

La actividad humana ha influenciado, en gran medida, el aumento considerable de gases de efecto invernadero en la atmósfera terrestre (Oreskes, 2004). Desde la segunda mitad del siglo XVIII y principios del XIX, con la revolución industrial, se inició un periodo de altas emisiones que ha generado profundos cambios en la temperatura media mundial, afectando, finalmente, la circulación atmosférica y, en consecuencia, la variabilidad climática. Estos cambios han despertado un creciente interés por parte de investigadores, de climatólogos, de agricultores, entre otros, debido a sus implicaciones sobre la mayoría de las actividades desarrolladas por el hombre, en especial, en la agricultura. El aumento en la temperatura modifica las fases fenológicas de la planta y ciclos cortos de altas o bajas temperaturas pueden afectar yemas florales, floración, número de granos, peso de granos y, con ello, los rendimientos (Chmielewski \& Rötzer, 2001; Chmielewski et al. 2004; McKeown et al. 2005).

Según el IDEAM (2009), el cambio en la periodicidad de los fenómenos El Niño - La Niña ha tenido un efecto importante en la cantidad y en la distribución de las lluvias, causando efectos negativos en la producción agrícola y pecuaria de amplias zonas continentales.

Las plantas cultivadas son sensibles a las variaciones del clima. Las temperaturas del aire cercanas al óptimo favorecen el crecimiento de la plantas, mientras que las bajas limitan de manera importante el crecimiento; temperaturas altas, de manera constante durante varios días, pueden ser muy perjudiciales, sobre todo si la humedad del suelo es baja. En las regiones semiáridas de África, donde se cultiva el maní, la frecuencia y la intensidad de las sequías han aumentado en los últimos 30 años (Hall et al. 2003), debido a los cambios climáticos, como número de días con precipitación y cantidad de precipitación por día y a las actividades humanas (Van Duivenbooden et al. 2002; Wittig et al. 2007). Esto ha provocado un cambio a cultivos más tolerantes a la sequía, como el caupí (Van Duivenbooden et al. 2002). Otros factores ambientales que intervienen de manera importante sobre el comportamiento fisiológico de las plantas son la luz y la cantidad de agua disponible (Wahid et al. 2007).

Al considerar lo anterior y, sobre la base de la importancia de la agricultura en la seguridad alimentaria de los pueblos (Deep, 2003), principalmente en los más vulnerables (Roudier et al. 2011) y las implicaciones futuras que el cambio climático tendría sobre la productividad (Soto, 2009), se presenta esta revisión con los conocimientos actuales acerca de los efectos del cambio climático sobre la fisiología de las plantas cultivadas, que cobra hoy más importancia ante el impacto negativo de este fenómeno en la agricultura (Nelson et al. 2009).

Variabilidad del clima: Esta característica, se refiere a las transiciones en las condiciones climáticas medias y otras estadísticas, como las desviaciones típicas, los fenómenos extremos, en todos los niveles temporales y espaciales, que se extienden más allá de la escala de un fenómeno meteorológico en particular. La variabilidad, se puede deber a procesos naturales internos que ocurren dentro del sistema climático (variabilidad interna), a variaciones en el forzamiento externo natural (astronómico) u otros forzamientos externos, como el antropógeno (variabilidad externa producida por el ser humano). Éste último invita a intervenir con instrumentos colectivos que permitan reducir el impacto humano sobre la biósfera (Elizalde, 2009). En síntesis, una oscilación natural y recurrente en el clima en escalas intra e interanual, como lo es una época lluviosa o seca en el año ocasionada por el paso de la ZCIT (Zona de Confluencia Intertropical) o generadas por los eventos El Niño (calentamiento del océano pacifico ecuatorial, central y oriental) o La Niña (enfriamiento del océano pacífico ecuatorial, central y oriental) en diversos años, son considerados como eventos de variabilidad climática (IDEAM, 2009).

Cambio climático: Actualmente, en la comunidad científica existe conocimiento de los mecanismos y de los procesos de cambio climático a distintas escalas temporales en el clima global y, lo que posiblemente sucederá en el próximo siglo, como resultado del aumento de concentraciones de gases de efecto invernadero, tales como el dióxido de carbono, metano, óxidos nitrosos, clorofluorocarbonos y vapor de agua (Houghton, 1985).

El cambio climático es una variación estadísticamente significativa, ya sea de las condiciones climáticas medias o de su 
variabilidad, que se mantiene durante un período prolongado (generalmente, durante decenios o por más tiempo). "La Convención Marco de las Naciones Unidas sobre el Cambio Climático (CMNUCC)", en su Artículo uno, define el cambio climático como: "cambio del clima atribuido directa o indirectamente a actividades humanas que alteran la composición de la atmósfera mundial, y que viene a añadirse a la variabilidad natural del clima observada durante períodos de tiempo comparables". La CMNUCC hace pues, una distinción entre "variabilidad del clima", atribuible a causas naturales y "cambio climático", atribuible a actividades humanas que alteran la composición de la atmósfera, como por ejemplo, el calentamiento global (IPCC, 2004).

El calentamiento global. El calentamiento global es el aumento progresivo de la temperatura media del planeta a lo largo del tiempo, ocasionado por el incremento de concentraciones de gases de efecto invernadero, cuyas concentraciones se han incrementado considerablemente desde 1750 (Tabla 1).

El efecto invernadero. La tierra recibe energía del sol en forma de radiación electromagnética y la superficie terrestre recibe radiación ultravioleta (onda corta) y radiación visible y emite radiación terrestre en forma de radiación infrarroja (onda larga). Estos dos grandes flujos energéticos deben estar en balance, pero la presencia de gases de efecto invernadero en la atmósfera afecta la naturaleza de este balance (IDEAM, 2009).

El efecto invernadero, se origina porque la energía que llega del sol, al proceder de un cuerpo de muy elevada temperatura, está formada por ondas de frecuencias altas que traspasan la atmósfera con gran facilidad. La energía remitida hacia el exterior desde la Tierra, al proceder de un cuerpo mucho más frío, está en forma de ondas de frecuencias más bajas y es absorbida por los gases con efecto invernadero. Esta retención de la energía hace que la temperatura sea más alta, aunque hay que entender bien que, al final, en condiciones normales, luego de aprovecharse parte en procesos biológicos y biofísicos, es igual la cantidad de energía que llega a la Tierra que la que esta emite. Si no fuera así, la temperatura de nuestro planeta habría ido aumentando continuamente, cosa que, por fortuna, no ha sucedido. Se podría decir, de una forma muy simplificada, que el efecto invernadero lo que hace es provocar que le energía que llega a la Tierra sea "devuelta" más lentamente, por lo que se "mantiene" más tiempo junto a la superficie y así eleva la temperatura (Echarri, 2011).

Los gases de efecto invernadero (dióxido de carbono, metano, óxidos nitrosos y clorofluorocarbonos, vapor de agua) permiten que la radiación de onda corta solar penetre sin impedimento, pero absorben la mayor parte de la emisión de onda larga terrestre. Por ello, la temperatura global promedio es de $14^{\circ} \mathrm{C}\left(57^{\circ} \mathrm{F}\right)$, pero podría ser tan baja como $-18^{\circ} \mathrm{C}\left(-0,4^{\circ} \mathrm{F}\right)$, es decir, $32^{\circ}$ más alto que si no tuviera atmósfera gaseosa. Este efecto se llama el "Efecto Invernadero" (GCCIP, 1997).

Estos gases están atrapando una porción creciente de radiación infrarroja terrestre y se espera que hagan aumentar la temperatura planetaria entre 1,5 y $4,5^{\circ} \mathrm{C}$. Como respuesta a esto, se estima que los patrones de precipitación global, también se alteren. Aunque existe un acuerdo general sobre estas conclusiones, existe una gran incertidumbre con respecto a las magnitudes y a las tasas de estos cambios a escalas regionales (EEI, 1997).

Tabla 1. Resumen de la variación en el contenido atmosférico de gases de efecto invernadero (Cambio Climático Global, 1997).

\begin{tabular}{|l|c|c|c|}
\hline Gas Invernadero & Concentración $\mathbf{1 7 5 0}$ & Concentración $\mathbf{1 9 9 2}$ & Fuerza Irradiativa (W/m²) \\
\hline Dióxido de Carbono & $\mathbf{2 8 0 ~ p p m v}$ & $355 \mathrm{ppmv}$ & 1,56 \\
\hline Metano & $0,8 \mathrm{ppmv}$ & $1,72 \mathrm{ppmv}$ & 0,5 \\
\hline Óxido Nitroso & $275 \mathrm{ppbv}$ & $310 \mathrm{ppbv}$ & 0,1 \\
\hline CFC-11 & 0 & $280 \mathrm{pptv}$ & (siguiente) \\
\hline CFC-12 & 0 & $484 \mathrm{pptv}$ & 0,3 (todos los CFCs) \\
\hline HCFCs/HFCs & 0 & Sin datos & 0,05 \\
\hline Ozono Troposférico & Sin datos & Variable & $0,2-0,6$ \\
\hline Ozono Estratosférico & Sin datos & 300 unidad dobson & $-0,1$ \\
\hline
\end{tabular}


Un panel de 2500 científicos de primera línea (IPCC, 2001) estuvo de acuerdo en que " ya se puede detectar un cambio discernible de influencia humana sobre el clima global entre las muchas variables naturales del clima». Según el panel, la temperatura de la superficie terrestre ha aumentado, aproximadamente, $0,6^{\circ} \mathrm{C}$, en el último siglo.

Consecuencias del cambio climático. De acuerdo con IPCC (2001, 2007), una duplicación de los gases de invernadero incrementaría la temperatura terrestre entre 1 y $4,5^{\circ} \mathrm{C}$. Aunque no parezca mucho es equivalente a volver a la última glaciación, pero en la dirección inversa. Por otro lado, el aumento de temperatura sería el más rápido en los últimos cien mil años, haciendo muy difícil que los ecosistemas del mundo se adapten (Watson, 2001).

Por otra parte, el aumento progresivo de la temperatura en la superficie terrestre ya ha mostrado sus efectos sobre la variabilidad climática, haciéndose cada vez más frecuentes y extremos los eventos meteorológicos y climáticos en el planeta (Mcgao, 2001). Otras corrientes científicas afirman que el llamado cambio climático es un periodo de transición de la tierra (ciclo o proceso natural del planeta), que puede durar miles o millones de años y que sin querer o no, tendría que suceder, restándole importancia al tema. Estos autores basan sus afirmaciones sobre la tesis que, en la actualidad, no hay una teoría concreta sobre la existencia y la comprobación de un cambio climático global (Stainforth et al. 2005; Svensmark, 2007; Schnellhuber, 2008).

Las fluctuaciones climáticas que se han venido registrando en diversas regiones de la tierra son objeto de estudio y preocupación, debido, principalmente, a su impacto en la producción de alimentos (Nelson et al. 2009). Hoy día, los agricultores presencian anomalías climáticas más intensas que las experimentadas años atrás; así, por ejemplo, algunos estudios prospectivos sobre el clima han estimado que la producción de café podría disminuir en un 30\%, para 2020 y hasta en un 70\%, para el 2050 (Gay \& Conde, 2004). Las variaciones de los ciclos del clima, con respecto a la normal, se traducen en pérdidas de cultivo (Jiménez et al. 2004). Por otra parte, el estrés por calor, debido al incremento de la temperatura, constituye un problema para la agricultura en muchas áreas del mundo.

Hay consenso en que el cambio climático, unido al incremento de la población, constituye una amenaza para la seguridad alimentaria en todas partes (Nelson et al. 2009). La agricultura es muy vulnerable al cambio climático, en particular, por los efectos del aumento de la temperatura en la germinación de las semillas de algunas especies (Carter \& Vavrina, 2001), causando desplazamientos de cultivos de áreas tropicales a zonas que antes eran frías o de áreas tem- pladas a zonas más frías (Jarvis et al. 2008), que terminará por reducir la producción y la calidad de los cultivos (Challinor et al. 2007; Tubiello et al. 2007b), a la vez, que provoca la proliferación de malas hierbas, artrópodos plaga y enfermedades. Los cambios en el régimen de lluvias aumentan las probabilidades de fracaso de las cosechas a corto plazo y de reducción de la producción a largo plazo (Nelson et al. 2009).

Cambio climático y fisiología de las plantas: El clima es uno de los principales factores que regulan la distribución de las especies de plantas, ya sea directamente, a través de limitaciones fisiológicas en el crecimiento y la reproducción o, indirectamente, por medio de los factores ecológicos, como la competencia por recursos (Shao \& Halpin, 1995). Diversos estudios destacan que el cambio climático registrado en los últimos 30 años ha tenido un impacto en la distribución, abundancia, fenología y fisiología de muchas especies (Osborn \& Briffa, 2005; Jarvis et al. 2008). Se presume que el incremento en la temperatura será entre 1,1 y $6,4^{\circ} \mathrm{C}$, para 2100 (IPCC, 2007), lo que de acuerdo con Thomas et al. (2004) y Jarvis et al. (2008) conducirá a la extinción de algunas especies del género Vigna ( $V$. longifolia y $V$. keraudrenii) y reducción del 63 al 100\% en el área de otras especies de este género. Por lo tanto, se generarán problemas de erosión genética, dificultando a futuro la solución de problemas limitantes de la producción agrícola. El mismo autor reporta importantes reducciones en los géneros Arachis y Solanum.

Se necesitan, entonces, cultivos y ganaderías que tengan éxito razonable en una gama relativamente amplia de condiciones de producción, en lugar de los que puedan tener en un conjunto limitado de condiciones climáticas (Nelson et al. 2009).

Para analizar los efectos del cambio climático sobre la fisiología de las plantas es necesario considerar varios componentes ambientales, que actúan de manera conjunta e interactiva sobre la expresión del vegetal. Por tal motivo y considerando que la mayoría de los estudios se refieren a los efectos individuales de las variables ambientales, se abordará la discusión desde el ángulo de cada factor, sin descuidar que la acción de éstos en la naturaleza se da en forma conjunta. Se discutirán los efectos de las variaciones ambientales relacionadas con los cambios en los niveles de $\mathrm{CO}_{2}$, temperatura, agua y ozono y sus interacciones sobre el crecimiento y desarrollo de las plantas.

Efecto de la temperatura sobre la fisiología de las plantas. De manera transitoria o constante, el estrés por calor causa cambios morfo-anatómicos, fisiológicos y bioquímicos en las plantas, que afectan su crecimiento y su desarrollo y puede conducir a una drástica reducción del rendimiento 
económico de las plantas cultivadas (Wahid et al. 2007). El aumento de la temperatura más allá del límite de tolerancia por un período de tiempo determinado causa daños irreversibles, a nivel celular, en cuestión de minutos (Schöffl et al. 1999), conduciendo a un colapso en la organización celular que incide en el crecimiento y en el desarrollo de plantas.

Este aumento de la temperatura hace que las zonas tropicales y subtropicales se conviertan, ante el cambio climático, en áreas limitantes para la producción de cultivos (Wahid et al. 2007) y amerita explorar e identificar, en la variabilidad genética existente, plantas con buenos atributos agronómicos, minerales y de adaptación a la nueva oferta ambiental. En tomate, temperaturas superiores a $34^{\circ} \mathrm{C}$, en regiones tropicales y subtropicales, cuando están asociadas con precipitaciones por encima de lo normal, conducen a bajas en los rendimientos, debido a la poca formación de flores, de gametos, de polinización, de fertilización y de desbalance de carbohidratos, así como el surgimiento de problemas fitosanitarios, por la aparición de marchitez bacteriana (Silva et al. 2000; Hanson et al. 2002). De igual manera, Craufurd et al. (2003), bajo condiciones controladas, evaluaron la sensibilidad de 22 cultivares de maní a temperaturas altas en floración, logrando identificar seis genotipos tolerantes y señalan que los estudios de modelación son de gran valor para identificar recursos genéticos asociados con tolerancia a altas temperaturas; por lo que la adaptación genotípica a los cambios en las temperaturas medias y extremas es relevante en la predicción de rendimientos (Challinor et al. 2007).

La germinación de semillas, se puede ver afectada por altas temperaturas $\left(>35^{\circ} \mathrm{C}\right)$, como sucede en tomate, en ají, en apio, en lechuga y en espinaca, lo cual, es conocido como termo-inhibición; sin embargo, la termo-tolerancia puede diferir entre cultivares de una misma especie, a causa del grado de actividad de la endo- $\beta$-mannanase en la semillas (Nascimento et al. 2000; Carter \& Vavrina, 2001).

El estrés por calor afecta el crecimiento de las plantas desde el principio de su ontogenia, aunque los efectos varían según la etapa de desarrollo. Las altas temperaturas pueden disminuir o inhibir totalmente la germinación de las semillas, dependiendo de las especies y de la intensidad del estrés; en etapas de desarrollo posteriores, las altas temperaturas pueden afectar adversamente la fotosíntesis, la respiración, las relaciones hídricas y la estabilidad de las membranas, así como los niveles de hormonas y de metabolitos secundarios. Además, la respuesta al estrés por calor está constituida por la producción de especies reactivas de oxígeno (ROS), de proteínas de choque térmico y otras proteínas relacionadas con el estrés que afectan el crecimiento y desarrollo de las plantas (Wahid et al. 2007).
En particular, en el algodonero (Gossypium hirsutum), se han realizado innumerables trabajos, con el propósito de evaluar los efectos de las temperaturas sobre su desarrollo fisiológico (Feller et al. 1998). Estos autores encontraron que la enzima Rubisco sufrió inhibición reversible a temperaturas superiores a $35^{\circ} \mathrm{C}$ y menores o iguales a $40^{\circ} \mathrm{C}$, pero a temperaturas mayores, la inhibición fue irreversible.

Algunos autores han ubicado a la Rubisco en ambientes con temperaturas altas y sugieren que su capacidad está, en principio, controlada por la tasa de fotosíntesis de especies con metabolismo $\mathrm{C}_{4}$ a bajas temperaturas (Kubien et al. 2003). Con base en sus resultados, estos autores proponen que la falta de éxito de las plantas $C_{4}$ en climas fríos refleja una limitación impuesta por tener menos Rubisco que las $C_{3}$, con las que compiten. Se puede pensar que ante los incrementos de temperatura, debido al cambio climático, aquellas especies $\mathrm{C}_{3}$ con menos Rubisco serán más susceptibles de desaparecer.

Reddy \& Pachepsky (2002) encontraron en el algodonero que a temperaturas mayores de $25^{\circ} \mathrm{C}$, la tasa de aparición de nudos y de elongación del tallo fue $40 \%$ más lenta, en tanto que la duración de expansión del tallo fue $23 \%$ más alta. Otros trabajos han demostrado que a bajas temperaturas $\left(20^{\circ} \mathrm{C}\right)$, se induce resistencia al frío y a los cambios en la composición de los ácidos grasos, mientras que a temperaturas altas $\left(40^{\circ} \mathrm{C}\right)$, se induce resistencia al calor y no se presenta alteración en la composición de los ácidos grasos (Rikin et al. 1993).

En otras especies, como el pasto de invierno, Poa annua, algunos investigadores han encontrado reducción de la materia seca con un aumento de $4^{\circ} \mathrm{C}$. Esto demuestra que la respuesta de los cultivos a los cambios de temperatura depende de la temperatura óptima de la fotosíntesis (Conroy et al. 1994). Cuando la temperatura está por debajo del óptimo para la fotosíntesis, un pequeño incremento puede estimular significativamente el crecimiento del cultivo (Wayne, 2002). A partir del valor óptimo de temperatura relacionado con el máximo rendimiento, un pequeño incremento puede reducir dramáticamente el mismo. La respuesta del cultivo a los incrementos en la temperatura también depende de la interacción con el aumento en la concentración de $\mathrm{CO}_{2}$. Altas temperaturas reducen las ganancias netas de carbono en especies $\mathrm{C}_{3}$ por incrementos en la fotorrespiración (Wayne, 2002).

Estudios de modelación realizados por Schlenker \& Lobell (2010) para diferentes regiones de África, donde evaluaron el impacto de los cambios de temperatura y de precipitación sobre el rendimiento, señalan que si las lluvias permanecen constantes, el rendimiento de los cultivos se reduce en un 
$15,0 \%$, en razón a que las altas temperaturas afectan el ciclo de los cultivos e incrementa el estrés hídrico, por la elevada evapotranspiración. En tanto, que el cambio en el régimen de precipitaciones puede agravar más la situación ante variaciones en la temperatura, dado que el aumento o reducción de las precipitaciones afecta los rendimientos entre $-10,0 \%$ y $-21,0 \%$, lo cual, concuerda con lo expresado por Izaurralde et al. (2003), quienes resaltan que la precipitación es el principal factor que afecta el rendimiento de los cultivos, ya que puede conducir a una reducción de biomasa.

Efecto de la radiación, la temperatura y la precipitación en las plantas. Los efectos del cambio climático sobre el rendimiento de los cultivos normalmente se presentan de manera independiente y la gran mayoría de los estudios consideran el efecto positivo y negativo del $\mathrm{CO}_{2}$. Hansen et al. (2002) y Mera et al. (2006) consideran que el estudio debe ser integral involucrando aspectos relacionados con el cambio climático: radiación, precipitación y temperatura, a fin de evaluar el impacto del cambio climático más allá del aumento del $\mathrm{CO}_{2}$ en la producción agrícola.

Mera etal. (2006), en un estudio de modelación, considerando la interacción de la radiación, temperatura y precipitación, en maíz y en soya, reportaron que el análisis simultáneo no coincide con los efectos individuales, especialmente, con la temperatura, donde el maíz resultó menos sensible que la soya. Los tres factores presentan un efecto no lineal sobre el rendimiento. Así mismo, la interacción de los tres factores puede reducir o alargar el ciclo de las plantas y dificulta el entendimiento de aspectos relacionados con la floración, llenado de grano y época de siembra; no obstante, la interacción radiación - precipitación contribuye significativamente al crecimiento de las plantas.

Efecto del $\mathrm{CO}_{2}$. Los trabajos realizados con el propósito de evaluar el efecto de niveles altos de $\mathrm{CO}_{2}$ sobre el cultivo del algodonero y de otras especies han sido numerosos y variados. La problemática se ha abordado desde el punto de vista genético, hormonal, de eficiencia fisiológica. Por ejemplo, la NASA -National Aeronautics and Space Administration, en 2001, informó que luego de un estudio desarrollado en el Sureste de Estados Unidos, que incluyó el norte de Florida, Georgia, Carolina del Norte, Carolina del Sur, Alabama, Mississippi, Louisiana, Arkansas y parte de Tennessee, los modelos utilizados predijeron incrementos en la producción del cultivo hasta de un 36\%, a gran escala, cuando los niveles de $\mathrm{CO}_{2}$ se duplicaron; sin embargo, estos estudios no consideraron factores ambientales adicionales, que interactúan ante un eventual aumento del $\mathrm{CO}_{2}$.

Parry et al. (2004) y Mera et al. (2006) sostienen que la respuesta relativa de las plantas $\mathrm{C}_{4}$ a las altas concentraciones de $\mathrm{CO}_{2}$ es pequeña comparada con las $\mathrm{C}_{3}$, dado el grado de saturación que se presenta en las $\mathrm{C}_{4}$; sin embargo, en un análisis a largo plazo, esto no siempre es verdadero para algunas especies silvestres $\mathrm{C}_{4}$ y las diferencias en respuesta al $\mathrm{CO}_{2}$ no son tan grandes (Stock et al. 2005). A este respecto, Ghannoum et al. (2000) y Kim et al. (2007) señalan que esto es verdaderamente cierto para el crecimiento, bajo condiciones ambientales no estresantes.

Yong et al. (2000) reportaron que altos niveles de $\mathrm{CO}_{2}$ incrementaron, significativamente, los contenidos de citoquininas en las hojas del algodonero con bajo contenido de nitrógeno (hojas viejas), mientras que en tejido foliar en crecimiento activo, el nivel de estas hormonas no varió al cambiar las concentraciones de $\mathrm{CO}_{2}$ ambiental.

Brunele et al. (2009) hallaron en tomate (Lycopersicon esculentum) que al enriquecer de manera intermitente la atmósfera con $\mathrm{CO}_{2}$, a concentraciones que fluctuaron entre 600 y 750 ppm, no se afectó la materia seca total de dos cultivares probados y apenas alteró, ligeramente, la distribución de masa seca de tallo en una de las variedades; en general, se observó un pequeña respuesta fisiológica de las plantas, con el enriquecimiento intermitente de $\mathrm{CO}_{2}$.

Tal vez esta respuesta obedece al hecho que el enriquecimiento con $\mathrm{CO}_{2}$ no fue constante, como lo anotan Calvert \& Slack (1975), quienes afirman que lo ideal es que las plantas de tomate estén sometidas durante todos los días a altas concentraciones de $\mathrm{CO}_{2}$, con lo cual, se obtiene una mejor respuesta fisiológica.

Efecto del $\mathrm{CO}_{2}$ y la temperatura sobre el desarrollo de las plantas. Lu (2005) evaluó un modelo denominado GOSSYM, que examina el impacto de los cambios de estas dos variables sobre el rendimiento y el requerimiento hídrico del algodón. El autor evaluó varios sitios productores y, aunque las respuestas son específicas para cada sitio, algunas tendencias generales indican que cuando la temperatura aumenta la producción declina y los requerimientos hídricos aumentan, por lo que los ingresos netos disminuyen. Por otro lado, cuando el nivel de $\mathrm{CO}_{2}$ se incrementa, la producción mejora, las necesidades de riego disminuyen y los ingresos netos aumentan. El impacto relativo de cambio de clima en la rentabilidad también varía entre las regiones, debido a las diferencias en los costos de producción (Nelson et al. 2009), especialmente, en el caso del agua.

En un trabajo realizado por Reddy \& Hodges (2005), bajo condiciones controladas, se evaluó el efecto de la variación de temperatura, $\mathrm{CO}_{2}$ y estado hídrico de las plantas. Los autores concluyeron varios aspectos importantes, tales como: a) la fotosíntesis neta fue menor bajo temperaturas 
mayores y menores que la óptima para el cultivo del algodón; este resultado se obtuvo sometiendo las plantas estudiadas a dos ambientes de $\mathrm{CO}_{2}$ y cinco temperaturas diferentes; b) a mayor temperatura se encontró una tasa mayor de abscisión de las cápsulas florales, tres a cinco días antes de la antesis; c) la doble concentración de $\mathrm{CO}_{2}$ indujo un incremento de la eficiencia fotosintética, debido a la intercepción de mayor luz en el dosel y, d) no hubo interacción significativa entre la respuesta fotosintética de plantas germinadas a alta y baja concentración de $\mathrm{CO}_{2}$ y condiciones de deficiencia hídrica. En ambos ambientes, las plantas disminuyeron sus tasas fotosintéticas cerca de un $43 \%$ al mediodía, cuando su potencial hídrico descendía de - 1,2 $\mathrm{MPa}$ a -3,0 MPa.

Efectos del déficit y el exceso hídrico sobre la fisiología de las plantas. La tolerancia a la sequía es definida como la capacidad de las plantas de vivir, de crecer y de obtener rendimientos satisfactorios bajo condiciones limitadas de agua en el suelo o en un período de estrés hídrico (Ashley, 1993) y los mecanismos que usan las plantas para hacer frente a la sequía, se agrupan en tres categorías, de acuerdo con Mitra (2001): escape, evitación y tolerancia; no obstante, las plantas pueden usar más de un mecanismo a la vez, para enfrentar la sequía.

Escape es la capacidad de la planta de completar su ciclo de vida antes que las condiciones de suelo presenten un déficit de agua, lo que implica un rápido desarrollo fenológico de la planta, plasticidad durante el período de desarrollo y removilización de asimilados, pero trae como consecuencia una reducción en los rendimientos, con respecto a las plantas de ciclo normal (Agbicodo et al. 2009).

Evitación, corresponde a la capacidad de las plantas de mantener las hojas turgentes, a pesar de la escasez de agua en el suelo, lo cual, se explica por una mayor profundidad de las raíces o un sistema de raíces eficiente en la absorción del agua, cierre de estomas, disminución de la absorción de la radiación o evapotranspiración por cierre de las hojas y reducida área foliar; ello repercute en una disminución en la asimilación de carbono, debido a la reducción de la transferencia física de moléculas de dióxido de carbono y el aumento de la temperatura foliar que favorece la reducción de los procesos bioquímicos y, por consiguiente, los rendimientos (Mitra, 2001).

El mecanismo de tolerancia implica que las plantas pueden resistir el déficit de agua en el suelo y se explica por el mantenimiento de la turgencia, a través de un ajuste osmótico, que implica acumulación de solutos en la célula, aumento de la elasticidad de las células, reducción del tamaño de las células y resistencia protoplasmática; sin embargo, se puede presentar una gran acumulación de iones por la pérdida de agua que, en altas concentraciones, se denomina estado vítreo, pudiendo causar desnaturalización de las proteínas y las membranas, ya que se incrementa la interacción entre moléculas (Agbicodo et al. 2009).

Durante la deshidratación, como consecuencia del estrés hídrico, se produce un incremento de las especies reactivas de oxígeno (ROS) (oxígeno singlete, radicales hidroxilo, peróxido de hidrógeno y aniones superóxido), en su mayoría en los cloroplastos y su efecto consiste en inhibir la reparación del daño al fotosistema II y la síntesis de la proteína D1 (Sunkar, 2010). Por otro lado, la sequía induce disminución de la actividad fotosintéticas estomatal y no estomatal e incremento en la actividad de las enzimas antioxidativas superóxido-dismutasa SOD, catalasa CAT, ascorbato-peroxidasa APX, glutatión-reductasa, además del aldehído malónico MDA, que contribuyen a la supervivencia bajo condiciones de sequía (Pompelli et al. 2010).

Reddy et al. (1997) observaron que las plantas de algodón que crecieron en condiciones deficientes de agua, en cámaras de ambiente controlado, disminuyeron sus tasas de fotosíntesis. Por otra parte, Ephrath et al. (2001) señalaron que el crecimiento de la raíz de algodón aumentó en plantas con estrés hídrico, comparado con los tratamientos con buen riego. El potencial hídrico en la hoja fue superior en el tratamiento con altos niveles de $\mathrm{CO}_{2}$, tanto en condiciones bien hidratadas como en condiciones de déficit hídrico.

Por otra parte, bajo condiciones de inundación, las tasas de asimilación de $\mathrm{CO}_{2}$ disminuyen drásticamente en especies sensibles a anoxia, como respuesta a este tipo de estrés; sin embargo, en especies tolerantes a inundación, como el álamo gris (Populum salba x Populus tremula), no resultan alteradas; incluso, después de una semana de hipoxia, el suministro de carbono reducido en forma de etanol, a través de la corriente de transpiración, se mantuvo alto (Kreuzwieser et al. 2009).

Efecto del $\mathrm{CO}_{2}$ sobre la transpiración y uso del agua por las plantas. La transpiración de los cultivos no responde tanto al incremento del $\mathrm{CO}_{2}$, como se ha predicho en mediciones foliares. El cierre estomático parcial reduce la tasa de transpiración y el flujo de calor latente, con aumento en la temperatura de la hoja; el resultado es un incremento en el gradiente que controla la pérdida de agua, lo cual, tiende a contrarrestar los efectos de cierre estomatal sobre la transpiración. Las altas temperaturas en el dosel y las reducciones en la transpiración son otra forma de retroalimentación del control estomatal de la transpiración. El déficit de presión de vapor del aire dentro e inmediatamente por encima de la vegetación, depende parcialmente de la transpiración. En la medida en que sea más lenta, tiende a secar el aire en los 
estratos del límite del dosel, con incremento del gradiente de presión de vapor (Wayne, 2002).

El agua es la variable crucial que más afecta la producción de algodón. Varios factores afectan el grado, en el cual, una reducción en la transpiración cambia la eficiencia en el uso de agua por unidad de área foliar. Si se considera que niveles elevados de $\mathrm{CO}_{2}$ causan cierre parcial de estomas es de esperarse un aumento de la temperatura de la hoja, al reducirse la termorregulación. La temperatura aumentada en el follaje incrementa la presión parcial de vapor de agua dentro de las hojas y aumenta la transpiración de la hoja. Al mismo tiempo, el aumento del crecimiento ante estímulos de $\mathrm{CO}_{2}$ altos ocasiona plantas con mayor área foliar, lo cual, tiende a aumentar la transpiración de la planta entera.

La temperatura del aire también afecta la tasa de transpiración. La tasa de transpiración de hojas individuales interactúa fuertemente con el $\mathrm{CO}_{2}$ y la temperatura, ya que se incrementó linealmente cuando la temperatura aumentó de 26 a $36^{\circ} \mathrm{C}$ (Reddy et al. 1998). A pesar de lo anterior, los autores recomiendan evitar extrapolar los efectos del $\mathrm{CO}_{2}$ sobre el dosel de las plantas, a partir de valores de hojas individuales.

Al nivel de la hoja, la eficiencia instantánea en el uso del agua o la eficiencia de transpiración (ET) puede ser definida como la relación entre la tasa de fotosíntesis neta o tasa de asimilación (A) y la transpiración (E) (Wayne, 2002). El valor de A en la hoja exhibe un típico incremento curvilíneo con el aumento del $\mathrm{CO}_{2}$, que continúa con altas concentraciones de este gas en especies con metabolismo $\mathrm{C}_{3}$ y $\mathrm{C}_{4}$ (Pearcy \& Ehleringer 1984). El metabolismo $\mathrm{C}_{4}$ concentra el $\mathrm{CO}_{2}$ en el sitio de fijación de la enzima ribulosa-1,5-bisfosfato carboxilasa/oxigenasa (Rubisco), indicando que la fotosíntesis en $\mathrm{C}_{4}$ es relativamente insensible a los incrementos internos de $\mathrm{CO}_{2}$ por encima de la concentración actual. Las altas temperaturas, por el contrario, reducen la fotosíntesis neta en plantas $\mathrm{C}_{3}$, debido al incremento en las pérdidas de carbono, que ocurre en el proceso de fotorrespiración (Wayne, 2002).

Efecto de la variación de $\mathrm{CO}_{2}$ y la nutrición mineral sobre el desarrollo de las plantas. Al relacionar el efecto de la variación del $\mathrm{CO}_{2}$ y la nutrición del algodonero, Reddy et al. (1997) indican que, si la planta presenta un estrés por deficiencias de agua, nitrógeno o potasio, la interacción de éste con el efecto del $\mathrm{CO}_{2}$ sobre la fotosíntesis es casi nula; sin embargo, los autores encontraron que la fotosíntesis declinó rápidamente durante el período de llenado de frutos cuando el nitrógeno fue deficiente, pero cuando los niveles de éste fueron adecuados la tasa de fotosíntesis del dosel, se mantuvo por encima del $50 \%$ al aumentar en dos veces el $\mathrm{CO}_{2}$, respecto a la misma situación con una condición de $\mathrm{CO}_{2}$ ambiental. Los autores informan, además, que la deficiencia de potasio causó una disminución entre $6,8 \%$ y $9,1 \%$ en la tasa de fotosíntesis en ambientes normales y duplicados de $\mathrm{CO}_{2}$, respectivamente. Por otra parte, la fotosíntesis fue casi nula en la planta cuando creció con una concentración de potasio, extremadamente baja.

Los estudios sobre el efecto de la fertilización con y sin $\mathrm{CO}_{2}$ sobre el rendimiento de los principales cultivos han registrado resultados contradictorios en los modelos utilizados por Müller et al. (2010). Al respecto, reportaron incrementos del $22 \%$ y decrecimientos del $13 \%$, argumentando que el aumento en el rendimiento está en función de un aumento en la tasa de asimilación de carbono así como en el uso eficiente del agua, según Tubiello et al. (2007a) Pero el incremento es incierto, ya que para ser aprovechable el carbono por el órgano de interés debe existir un suministro de nutrientes de acuerdo a su demanda.

Por otro lado, la elevada fertilización con $\mathrm{CO}_{2}$ puede afectar la calidad nutricional, por una reducción del contenido de proteína (Taub et al. 2008) o la susceptibilidad a malezas, enfermedades e insectos (Bale et al. 2002; Dermody et al. 2008; Zavala et al. 2008; Olesen et al. 2011).

Parry et al. (2004) y Roudier et al. (2011) reportaron en sus simulaciones relacionadas con el efecto de la fertilización, con y $\sin \mathrm{CO}_{2}$, resultados contrastantes con respecto a Müller et al. (2010), entre los años 2020 y 2080, anotando Roudier et al. (2011), que ello depende del tipo de cultivo, horizonte de tiempo y escenario de emisiones.

Efecto del ozono sobre el desarrollo de las plantas. Numerosos estudios han demostrado que el ozono $\left(\mathrm{O}_{3}\right)$ reduce el crecimiento de las plantas y cambia la partición de los asimilados. El patrón de dichos cambios varía con las especies, pero tiende a sugerirse un modelo general. $\mathrm{El}_{3}$, generalmente, reduce la cantidad de materia seca en toda la planta.

Cooley \& Manning (1987) indican que este gas puede reducir el número de flores o semillas, pero éstas con frecuencia tienen una acumulación total de materia seca, comparable a las de plantas que no han sido sometidas al mismo estrés. A niveles altos de $\mathrm{O}_{3}$, la acumulación de asimilados se reduce considerablemente; no obstante, es significativo que los órganos de almacenamiento, aquellos que suministran energía para el nuevo crecimiento en plantas perennes, como los árboles, son los más afectados por los cambios en la partición de asimilados inducida por el ozono, cuando sus concentraciones están en un rango que es común en ambientes contaminados.

Grantz \& Yang (1996) evaluaron el efecto del $\mathrm{O}_{3}$ sobre la arquitectura hidráulica en algodones Pima, resaltando que 
altos niveles de $\mathrm{O}_{3}$ reducen la eficiencia hidráulica radical en todas las plantas, con disminución de intercambio de gases y producción de biomasa. Por su parte, Grantz et al. (2003) también reportan una disminución en la actividad de la fuente. Así mismo, Grantz \& Farrar (1999) encontraron que el volumen de los carbohidratos transportados desde las hojas fuente se redujo por efectos del ozono, tanto en la asimilación como en el flujo, con un impacto directo sobre la translocación, a través del floema. Este rápido flujo cinético, probablemente, se refleja en un daño oxidativo al plasmalema o plasmodesmos del mesófilo o a células acompañantes del floema.

En otras especies, como calabaza, Castagna et al. (2001) reportaron que en hojas jóvenes y maduras, el $\mathrm{O}_{3}$ indujo una disminución significativa en la tasa fotosintética por impactos sobre las estomas y la fotoquímica. Por otro lado, en hojas de Phaseolus vulgaris var. Pinto, expuestas a 0,10 ppm (196 micra $\mathrm{g} / \mathrm{m}^{3}$ ) de ozono a 15,24 y $32^{\circ} \mathrm{C}$, el desarrollo del daño foliar fue de 25,3 y $24 \%$, respectivamente. La conductancia estomática de hojas expuestas y no expuestas fue menor en la condición de $15^{\circ} \mathrm{C}$, intermedia a $24^{\circ} \mathrm{C}$ y mayor al crecer a $32^{\circ} \mathrm{C}$. La exposición al ozono redujo significativamente la difusión estomática en la condición de $32^{\circ} \mathrm{C}$, pero no a 24 o $15^{\circ} \mathrm{C}$ (Miller \& Davis, 1981).

De lo expuesto en esta revisión, se concluye que el cambio climático registrado en los últimos 30 años ha tenido un impacto en la distribución, la abundancia, la fenología y la fisiología de muchas especies y se presume un incremento en la temperatura de 1,1 a $6,4^{\circ} \mathrm{C}$, para 2100 , con consecuencias de erosión genética, extinción de muchas especies vegetales e inseguridad alimentaria.

El estrés por calor causa cambios morfo-anatómicos, fisiológicos y bioquímicos en las plantas, que afectan su crecimiento y su desarrollo y pueden conducir a una drástica reducción del rendimiento económico de las plantas cultivadas.

Las altas temperaturas pueden disminuir o inhibir totalmente la germinación de polen, de semillas y afectar adversamente la fotosíntesis, la respiración, las relaciones hídricas, la estabilidad de las membranas, la regulación hormonal y, en general, el metabolismo de las plantas.

El estrés hídrico conduce a un incremento de las especies reactivas de oxígeno (ROS) (oxígeno singlete, radicales hidroxilo, peróxido de hidrógeno y aniones superóxidos), que causan daños al sistema fotosintético de las plantas y, simultáneamente, se produce un incremento en la actividad de las enzimas antioxidativas super-óxido-dismutasa SOD, catalasa CAT, ascorbato-peroxidasa APX, glutatión-reductasa, además del aldehído malónico MDA, que contribuyen a la disminución de los daños para la supervivencia, bajo tales condiciones.

La inundación induce disminución de la tasas de asimilación de $\mathrm{CO}_{2}$, sobre todo en especies sensibles a anoxia, con disminución del suministro de carbono reducido en forma de etanol en la corriente de transpiración.

La exposición al ozono $\left(\mathrm{O}_{3}\right)$ reduce la difusión estomática, la tasa fotosintética y afecta la partición de asimilados y, en general, reduce el crecimiento de las plantas.

Conflicto de intereses: El manuscrito fue preparado y revisado con la participación de todos los autores, quienes declaramos que no existe ningún conflicto de interés que ponga en riesgo la validez de los resultados presentados.

\section{BIBLIOGRAFÍA}

1. AGBICODO, E.M.; FATOKUN, C.A.; MURANAKA, S.; VISSER, R.G.F.; LINDEN VAN DER, C.G. 2009. Breeding drought tolerant cowpea: constraints, accomplishments, and future prospects. Euphytica (Holanda). 167(3):353-370.

2. ASHLEY, J. 1993. Drought and crop adaptation. In: Rowland, J.R.J. (ed) Dryland farming in Africa. Macmillan Press Ltd, UK. p.46-67.

3. BALE, J.S.; MASTERS, G.J.; HODKINSON, I.D.; AWMACK, C.; BEZEMER, T.M.; BROWN, V.K.; BUTTERFIELD, J.; BUSE, A.; COULSON, J.C.; FARRAR, J.; GOOD, J.E.G.; HARRINGTON, R.; HARLEY, S.; JONES, T.H.; LINDROTH, R.L.; PRESS, M.C.; SYMRNIOUDIS, I.; WATT, A.D.; WHITTAKER, J.B. 2002. Herbivory in global climate change research: direct effects of rising temperature on insect herbivores. Global Change Biol.(Inglaterra). 8(1):1-16.

4. BRUNELE, F.; DA SILVA, D.; PEDROSA, D.; CARDOSO,T.; PUIGNAL, A. 2009. Enriquecimento intermitente de $\mathrm{CO} 2$ e análise do acúmulo de massa seca e da partição de fotoasimilados em tomateiro. Acta Agr. (Colombia). 58(3):133-139.

5. CALVERT, A.; SLACK, G. 1975. Effects of Carbon dioxide enrichment on growth, development and yield of glasshouse tomatoes. Responses to controlled concentrations. Hort. Sci. (Estados Unidos). 50(1):61-71.

6. CAMBIO CLIMÁTICO GLOBAL.COM. 1997. Lista resumen sobre gases invernadero. Disponible desde 
Internet en http://www.cambioclimaticoglobal.com/ gasesinv (con acceso 26/03/2011).

7. CARTER, A.K.; VAVRINA, CH. S. 2001. High Temperature inhibits germination of jalapeño and cayenne Pepper. Hort. Sci. 36(4):724-725.

8. CASTAGNA, A.; NALI, S.; CIOMPI, G.; LORENZINI, G.; SOLDATINI, F.; RANIERI, A. 2001. Ozone exposure affects photosynthesis of pumpkin (Cucurbita pepo) plants. New Phytologist (Inglaterra). 152(2):223-229.

9. CONROY, J.P.; SENEWEERA, S.; BASRA, A.S.; ROGERSAND, G.; NISSEN, B. 1994. Influence of rising atmospheric $\mathrm{CO}_{2}$ concentrations and temperature on growth, yield and grain quality of cereal crops. Aus. J. Plant Physiol. 21(6):741-758.

10. COOLEY, D.; MANNING, W.J. 1987.The impact of ozone on assimilate partitioning in plants: A review. Environm. Poll. (EstadosUnidos). 47(2):95-113.

11. CRAUIFURD, P.Q.; PRASAD, P.V.V.; KAKANI, V.G.; WHEELER, T.R.; NIGAM, S.N. 2003. Heat tolerance in groundnut. Field Crops Res. (Estados Unidos). 80(1):63-77.

12. CHALLINOR, A.J.; WHEELLER, T.R.; CRAUIFURD, P.Q.; FERRO, C.A.T.; STEPHENSON, D.B. 2007. Adaptation of crops to climate change through genotypic responses to mean and extreme temperatures. Agr., Ecosyst. Environm. (Estados Unidos). 119(1-2):190-204.

13. CHMIELEWSKI, F.M.; RÖTZER, T. 2001. Response of tree phenology to climate change across. Europ. Agr. Forest Meteorology (Canadá). 108(1):101-112.

14. CHMIELEWSKI, F.M.; MÜLLER, A.; BRUNS, E. 2004. Climate changes and trends in phenology of fruit trees and field crops in Germany, 1961-2000. Agr. Forest Met.121(1):69-78.

15. DEEP, F. 2003. Enfoques conceptuales y sistemas de información sobre seguridad alimentaria. Ponencia realizada para el seminario de políticas de seguridad alimentaria y nutrición en América Latina. FODEPAL, Campinas (Brasil), 13p.

16. DERMODY, O.; O'NEILL, B.F.; ZANGERL, A.R.; BERENBAUM, M.R.; DELUCIA, E.H. 2008. Effects of elevated $\mathrm{CO}_{2}$ and $\mathrm{O}-3$ on leaf damage and insect abun- dance in a soybean agroecosystem. Arthropod-Plant Interactions (Finlandia). 2(3):125-135.

17. ECHARRI, P.L. 2011. Ciencias de la Tierra y del Medio Ambiente. Libro electrónico.2011. Disponible desde Internet en: http://www.tecnun.es/asignaturas/ecologia/ (con acceso 26/03/11).

18. EEI (Estación Espacial Internacional). 1997. La convención marco sobre cambio climático Introducción. Disponible desde Internet en: http://www.cambioclimaticoglobal.com (con acceso 24/03/11).

19. ELIZALDE, A. 2009. ¿Qué desarrollo puede llamarse sostenible en el siglo XXI? Universidad Bolivariana Santiago de Chile. Revista de Educación. Número extraordinario. p.53-75. Disponible desde Internet en: www.revistaeducacion.mec.es. (con acceso 26/07/2011).

20. EPHRATH, J.; TIMLIN, D.J.; BAKER, J.T.; REDDY, V. 2001. Effects of elevated $\mathrm{CO}_{2}$ concentration and water stress on the root to shoot ratio of cotton. Abstract of ASA-CSSA-SSSA International Annual Meeting. Disponible desde Internet en: http://cottondb.org/ cgi-bin/ace/tree/cottondb?name =ACS-2001-c02ephrath080054-P\&class $=$ Reference $\quad($ con acceso 26/07/2011).

21. FELLER, U.; CRAFTS-BRANDNER, S.; SALVUCCI, Y.M. 1998. Moderately high temperatures inhibit ribulose-1,5-bisphosphate carboxylase/oxygenase (rubisco) activase-mediated activation of Rubisco. Plant Physiol. (Estados Unidos). 116(2):539-546.

22. GAY, C.; CONDE, C. 2004. El cambio climático y el café. Bol. Inform. El Faro. UNAM. p.8-9.

23. GCCIP (Global Climate Change Information Programme). 1997. La convención marco sobre cambio climático. El presupuesto energético de la atmósfera. Disponible desde Internet en: http://www. cambioclimaticoglobal.com/presupue.html (con acceso 25/03/11).

24. GHANNOUM, O.; VON CAEMMERER, S.; ZISKA, L.H.; CONROY, J.P. 2000. The growth response of C4 plants to rising atmospheric $\mathrm{CO}_{2}$ partial pressure: a reassessment. Plant, Cell Environm. (Estados Unidos). 23(9):93-942.

25. GRANTZ D.; FARRAR, J. 1999. Acute exposure to ozone inhibits rapid carbon translocation from source 
leaves of Pima cotton. J. Experim. Botany (Reino Unido). 50(336):1253-1262.

26. GRANTZ, D.; YANG, S. 1996. Effect of $\mathrm{O}_{3}$ on hydraulic architecture in Pima cotton (biomass allocation and water transport capacity of roots and shoots). Plant Phys. 112(4):1649-1657.

27. GRANTZ, D.; SILVA, V.; TOYOTA, M.; OTT, N. 2003. Ozone increases root respiration but decreases leaf $\mathrm{CO}_{2}$ assimilation in cotton and melon. J. Exp. Botany. 54(391):2375-2384.

28. HALL, A.E.; CISSE, N.; THIAW, S.; ELAWAD, H.O.A.; EHLERS, J.D.; ISMAIL, A.M.; FERY, R.; ROBERTS, P.A.; KITCH, L.W.; MURDOCK, L.L.; BOUKAR, O.; PHILLIPS, R.D.; MCWATTERS, K.H. 2003. Development of cowpea cultivars and germplasm by the Bean/Cowpea CRSP. Field Crops Res. 82:103-134.

29. HANSEN, J.; SATO, M.; NAZARENKO, L.; RUEDY, R.; LACIS, A.; KOCH, D.; TEGEN, I.; HALL, T.; SHINDELL,D.; STONE, P.; NOVAKOV, T.; THOMASON, L.; WANG, R.; WANG,Y.; JACOB, D.J.; HOLLANDSWORTH-FRITH, S.; BISHOP, L.; LOGAN, J.; THOMPSON, A.; STOLARSKI, R.; LEAN, J.; WILLSON, R.; LEVITUS, S.; ANTONOV, J.; RAYNER, N.; PARKER, D.; CHRISTY, J. 2002. Climate forcings in the Goddard Institute for Space Studies SI2000 simulations. J. Geophys. Res. (Estados Unidos). 107(D18):4347-4384.

30. HANSON, P.M.; CHEN, J.; KUO, G. 2002. Gene action and heritability of high temperature fruit set in tomato line CL5915. Hort Sci. 37(1):172-175.

31. HOUGHTON, D. 1985. Handbook of Applied Meteorology. John Wiley \& Sons. New York, Chapter 40. Environmental impacts. I. Van der Hoven. p.11471163. Disponible desde Internet en: http://www. mma.es (con acceso 23/03/2011).

32. HUNT, B.G.; ELLIOTT, T. 2002. Mexican mega drought. Climate Dynamics (Alemania). 20(1):1-12.

33. IDEAM (Instituto de Hidrología, Meteorología y Estudios Ambientales, CO). 2009. Los fenómenos el niño / la niña. Disponible desde Internet en: http://www. ideam.gov.co (con acceso 23/03/2011).

34. IPCC (Intergovernmental Panel of Climate Change). 2001. Climate Change 2001: Volume II Impacts,
Adaptation and Vulnerability, Cambridge University Press. 397p.

35. IPCC (Intergovernmental Panel of Climate Change). 2004. Describing Scientific Uncertainties in Climate Change to Support Analysis of Risk and of Options. Disponible desde Internet en: http://www.ipcc.ch/pdf/ supporting-material/ipcc-workshop-2004-may.pdf (con acceso 25/03/2010).

36. IPCC (Intergovernmental Panel of Climate Change). 2007. Climate change 2007: Synthesis report. Summary for policymakers. Disponible desde Internet en: http://www.ipcc.ch. (con acceso 25/03/11).

37. IZAURRALDE, R.C.; ROSENBERG, N.J.; BROWN, R.A.; THOMSON, A.M. 2003. Integrated assessment of Hadley Center (HadCM2) climate-change impacts on agricultural productivity and irrigation water supply in the conterminous United States Part II. Regional agricultural production in 2030 and 2095. Agr. Forest Meteorology.117(1-2):97-122.

38. JARVIS, A.; LANE, A.; HIJMANS, R. 2008. The effect of climate change on crop wild relatives. Agr., Ecosyst. Environm. 126(1):13-23.

39. JIMÉNEZ L., T.; MORALES A., T.; REYNA T., V.; HERNÁNDEZ M., S.; OROZCO, F.; LEDESMA G., J. 2004. Dinámica de la sequía intra-estival en el estado de Tlaxcala, México". III Seminario Latinoamericano de Geografía Física, 28 abril- 2 mayo. (CDROM). Puerto Vallaría, Jalisco, México, memorias del evento. p.52-64.

40. KIM, S.H.; GITZ, D.C.; SICHER, R.C.; BAKER, J.T.; TIMLIN, D.J.; REDDY, V.R. 2007. Temperature dependence of growth, development, and photosynthesis in maize under elevated $\mathrm{CO}_{2}$. Environm. Exp. Botany. 61(3):224-236.

41. KREUZWIESER, J.; HAUBERG, J.; HOWELL, K.; CARROLL, A.; RENNENBERG, H.; MILLAR, H.; WHELAN, J. 2009. Differential response of gray poplar leaves and roots underpins stress adaptation during hypoxia. Plant Physiol. 149(1):461-473.

42. KUBIEN, D.; VON CAEMMERER, S.; FURBANK, R.; SAGE, R. 2003. $C_{4}$ photosynthesis at low temperature. A study using transgenic plants with reduced amounts of Rubisco. Plant Physiol. 132(3):1577-1585. 
43. LAU, CH.; JARVIS, A.; RAMÍREZ, J. 2011. Agricultura Colombiana: Adaptación al Cambio Climático. Centro Internacional de Agricultura Tropical (CIAT). $4 p$.

44. LU, YAO-CHI. 2005. An Economic Analysis of Climate Change on Cotton Production Across the U.S. Cotton Belt. Disponible desde Internet en: http://www. ars.usda.gov/research/publications/Publications. (con acceso 06/10/2005).

45. MCGAO. 2001. La simulación del sistema climático y sus cambios. Disponible desde Internet en: http://www. grida.no/climate/ipcc_tar (con acceso 23/03/2011).

46. MCKEOWN, A.; WARLAND, J.; MCDONALD, M.R. 2005. Long-term marketable yields of horticultural crops in southern Ontario in relation to seasonal climate. Can. J. Plant Sci. 85(2):431-438.

47. MERA, R.J.; NIYOGI, D.; BUOL, G.; WILKERSON, G.; SEMAZZI, F. 2006. Potential individual versus simultaneous climate change effects on soybean (C3) and maize (C4) crops: An agrotechnology model based study. Global and Planetary Change (Reino Unido). 54(1-2):163-182.

48. MILLER, C.; DAVIS, D. 1981. Effect of temperature on stomatal conductance and ozone injury of pinto bean leaves. Plant Disease. 65(9):750-751.

49. MITRA, J. 2001. Genetics and genetic improvement of drought resistance of crop plants. Current Sci. (India). 80(6):758-763.

50. MÜLLER, C.; BONDEAU, A.; POPP, A.; WAHA, K.; FADER, M. 2010. Climate change impacts on agricultural yields. Background note to the World Development Report 2010. Potsdam Institute for Climate Impact Research, Potsdam.12p. Disponible desde Internet en: www.pik-potsdam.de/research (con acceso 28/08/2011).

51. NASA (NATIONAL AERONAUTICS AND SPACE ADMINISTRATION). 2001. Cotton doesn't shrink from climate change. Disponible desde Internet en: $\quad$ http://www.gsfc.nasa.gov/news-release/ releases/2001/01-118.htm (con acceso 06/10/2005).

52. NASCIMENTO, W.M.; CANTLIFFE, D.J.; HUBER, D.J. 2000. Thermotolerance in lettuce seeds: Association with ethylene and endo- $\beta$-mannanase. J. Amer. Soc. Hort. Sci.125 (4):518-524.
53. NELSON, C.; ROSEGRANT, M.; KOO, J.; ROBERTSON, R.; SULSER, T.; ZHU, T., RINGLER, C.; MSANGI, S.; PALAZO, A., BATKA, M., MAGALHAES, M.; VALMONTE-SANTOS, R.; EWING, M., LEE, D. 2009. Cambio climático: el impacto en la agricultura y los costos de adaptación. Instituto Internacional de Investigación sobre políticas alimentarias IPFRI. 30p. Disponible desde Internet en: www.fao.org. (con acceso 10/08/2010).

54. NRDC (Natural Resources Defense Council). 2008. La onda verde; Datos principales sobre el calentamiento global. Disponible desde Internet en: http://www. nrdc.org/laondaverde/globa (con acceso 21/03/ 2011).

55. OLESEN, J.E.; TRNKAB, M.; KERSEBAUMC, K.C.; SKJELVAGD, A.O.; SEGUINE, B.; PELTONENSAINIOF, P.; ROSSIG,F.; KOZYRAH, J.; MICALEI, F. 2011. Impacts and adaptation of European crop production systems to climate change. Europ. J. Agron. (Francia). 34(1):96-112.

56. ORESKES, N. 2004. Beyond the Ivory Tower. The Scientific Cosensus on Climatic Change. Science. 306(5702):1686.

57. OSBORN, T.J.; BRIFFA, K.R. 2005. The spatial extent of 20th-century warmth in the context of the past 1200 years. Science. 311(5762):841-844.

58. PARRY, M.L.; ROSENZWEIG, C.; IGLESIAS, A.; LIVERMORE, M.; FISCHER, G. 2004. Effects of climate change on global food production under SRES emissions and socioeconomic scenarios. Global Environm. Change (ReinoUnido).14(1):53-67.

59. PEARCY, R.W.; EHLERINGER, J. 1984. Comparative ecophysiology of $\mathrm{C}_{3}$ and $\mathrm{C}_{4}$ plants. Plant, Cell Environm. 7(1):1-13.

60. POMPELLI, M.; BARATA, R.; VITORINO, H.; GONÇALVES, E.; ROLIM, E.; SANTOS, M.; ALMEIDA, J.; FERREIRA, V.; LEMOS, E.; ENDRES, L. 2010. Photosynthesis, photoprotection and antioxidant activity of purging nut under drought deficit and recovery. Biomass Bioenergy (ReinoUnido). 34(8):1207-1215.

61. REDDY, K.; HODGES, H. 2005. Impacts of climate change on cotton production: a Southcentral assessment. Disponible desde Internet en: http://nigec. ucdavis.edu/ (con acceso el 06/10/2005). 
62. REDDY, K.; HODGES, H.F.; MCKINION, J.M. 1997. Water and nutrient deficits, crop yields and climate change. World Resource Rev. (Estados Unidos). 10(1):23-43.

63. REDDY, K.; REDDY, R.; HODGES, H.F. 1998. Interactive effects of elevated carbon dioxide and growth temperature on photosynthesis in cotton leaves. Plant Growth Reg. Surafrica). 26(1):33-40.

64. REDDY, V.; PACHEPSKY, Y.Y. 2002. Temperature effects on node development rates in cotton. Ann. Botany (EstadosUnidos). 31:101-111.

65. RIKIN, A.; DILLWITH, J.W.; BERGMAN, D.K. 1993. Correlation between the circadian rhythm of resistance to extreme temperatures and changes in fatty acid composition in cotton seedlings. Plant Physiol. 101(1):31-36.

66. ROUDIER, P.; SULTAN, B.; QUIRION, P.; BERG, A. 2011.The impact of future climate change on West African crop yields: What does the recent literature say? Global Environm. Change. 21(3):1073-1083.

67. SCHLENKER, W.; LOBELL, D.B. 2010. Robust negative impacts of climate change on African agriculture. Environm. Res. Letters (Reino Unido). 5(1):1-8.

68. SCHNELLHUBER, H. 2008. Global warming: Stop worrying, start panicking? PNAS (Estados Unidos). 105(38):14239-14240.

69. SCHÖFFL, F.; PRANDL, R.; REINDL, A. 1999. Molecular responses to heat stress. In: Shinozaki, K., Yamaguchi-Shinozaki, K. (Eds.). Molecular Responses to Cold, Drought, Heat and Salt Stress in Higher Plants. R.G. Landes Co., Austin, Texas, p.81.98.

70. SHAO, G.; HALPIN, P.N. 1995. Climatic controls of eastern North American coastal tree and shrub distributions. J. Biogeography (Estados Unidos). 22 (6):1083-1089.

71. SILVA, A.C.; LEITE, I.C.; BRAZ, L.T. 2000. Avaliação da viabilidade do pólen como possível indicativo de tolerância a altas temperaturas em genótipos de tomateiro. Rev. Bras. Fisiol. Vegetal.12(2):156-165.

72. SOTO, W. 2009. La teoría de la sociedad del riesgo global: cंes capaz de dar cuenta del acontecer mundial actual? Bol. Observatorio Política Exterior (Costa Rica). 3(11):8-12.
73. STAINFORTH, D.; AINA, T.; CHRISTENSEN, C.; COLLINS, M.; FAULL, N.; FRAME, D.; KETTLEBOROUGH J.; KNIGHT, S.; MARTI, A.; MURPHY, J.; PIANI, C.; SEXTON, D., SMITH, L.; SPICER, R.; THORPE, A.; ALLEN, M. 2005. Uncertainty in predictions of the climate response to rising levels of greenhouse gases. Nature:433 (7024):403-406.

74. STOCK, W.D.; LUDWIG, F.; MORROW, C.D.; MIDGLE, G.F.; WAND, S.J.E.; ALLSOPP, N.; BELL, T.L..2005. Long-term effects of elevated atmospheric $\mathrm{CO}_{2}$ on species composition and productivity of a southern African $\mathrm{C}_{4}$ dominated grassland in the vicinity of a $\mathrm{CO}_{2}$ exhalation. Plant Ecol. (Australia).178(2):211-224.

75. SUNKAR, R. 2010. Plant Stress Tolerance.Methods and Protocols. Oklahoma state University USA. Humana Press. 401p.

76. SVENSMARK, H. 2007. Cosmoclimatology: a new theory emerges. Astronomy \& Geophysics (Reino Unido). 48(1):18-24.

77. TAUB, D.R.; MILLER, B.; ALLEN, H. 2008. Effects of elevated $\mathrm{CO}_{2}$ on the protein concentration of food crops: a meta-analysis. Global Change Biol. 14(3):565-575.

78. THOMAS, C.D.; CAMERON, A.; GREEN, R.E.; BAKKENES, M.; BEAUMONT, L.J.; COLLINGHAM, Y.C.; ERASMUS, B.F.N.; FERREIRA DE SIQEIRA, M.; GRAINGER, A.; HANNAH, L.; HUGHES, L.; HUNTLEY, B.; VAN JAARSVELD, A.S.; MIDGLEY, G.F.; MILES, L.; ORTEGA-HUERTAS, M.A.; PETERSON, A.T.; PHILLIPS, O.L.; WILLIAMS, S.E. 2004. Extinction risk from climate change. Nature. 427(6970):145-148.

79. TUBIELLO, F.N.; JAMTHOR, J.S.; BOOTE, K.J.; DONATELLI, M.; EASTERLING, W.; FISCHER, G.; GIFFORD, R.M.; HOWDEN, M.; REILLY, J.; ROSENZWEIG, C. 2007a. Crop response to elevated $\mathrm{CO}_{2}$ and world food supply - A comment on "Food for Thought..." by Long et al., Science. 312:1918-1921, 2006. Europ. J. Agron. 26(3):215-223.

80. TUBIELLO, F.N.; SOUSSANA, J.F.; HOWDEN, S.M. 2007b. Crop and pasture response to climate change.PNAS.Disponible desde internet en:http:// www.pnas.org/cgl/ddl/10.1073/pnas.0701728104.

81. VAN DUIVENBOODEN, N.; ABDOUSSALAM, S.; MOHAMED, A.B. 2002. Impact of climate change on 
agricultural production in the Sahel-Part 2. Case study for groundnut and cowpea in Niger. Climatic Change 54(3):349-368.

82. WAHID, A.; GELANI, S.; ASHRAF, M.; FOOLAD, M.R. 2007. Heat tolerance in plants: An overview. Environm. Exp. Botany. 61(3):199-223.

83. WATSON, R. 2001. Evaluación del Grupo Intergubernamental de Expertos sobre el Cambio Climático (IPCC). Disponible desde Internet en: http://www. ipcc.ch/pub/un/syrspanish/spm.pdf (con acceso 24/03/2011).

84. WAYNE, H. 2002. Implications of atmospheric and climatic change for crop yield and water use efficiency. Crop Sci.(Estados Unidos). 42(1):131140.
85. WITTIG, R.; KÖNIG, K.; SCHMIDT, M.; SZARZYNSKI, J. 2007. A study of climate change and anthropogenic impacts in West Africa. Environm. Sci. Poll. Res. 14(3):182-189.

86. YONG, J.; WONG, CH.; LETHAM, D.; HOCART, CH.; FARQUHAR, G. 2000. Effects of elevated $\left[\mathrm{CO}_{2}\right]$ and nitrogen nutrition on cytokinins in the xylem sap and leaves of cotton. Plant Physiol. (Estados Unidos).124(2):767-780.

87. ZAVALA, J.A.; CASTEEL, C.L.; DELUCIA, E.H.; BERENBAUM, M.R. 2008. Anthropogenic increase in carbon dioxide compromises plant defense against invasive insects. Proc. Nal Acad. Sci. 105:5129-5133.

Recibido: Diciembre 12 de 2011

Aceptado: Abril 9 de 2012 